\title{
Research on the Dynamic Model of Entrepreneurship Based on Improved Machine Learning
}

\author{
Mengbin Zhu, ${ }^{1}$ Yan Yang, ${ }^{1}$ and Huaying Cao $\mathbb{D}^{2}$ \\ ${ }^{1}$ The Open University of Shaanxi, Xi'an 710119, China \\ ${ }^{2}$ Zhengzhou Shengda University, Zhengzhou 451191, China \\ Correspondence should be addressed to Huaying Cao; 100740@shengda.edu.cn
}

Received 13 August 2021; Revised 10 September 2021; Accepted 29 September 2021; Published 23 October 2021

Academic Editor: Mu Zhou

Copyright (C) 2021 Mengbin Zhu et al. This is an open access article distributed under the Creative Commons Attribution License, which permits unrestricted use, distribution, and reproduction in any medium, provided the original work is properly cited.

\begin{abstract}
There are many influencing factors in the entrepreneurial process, which lead to a variety of unknown situations in the entrepreneurial process and affect the entrepreneurial process. In order to improve the effect of entrepreneurial analysis, this paper improves the traditional machine learning algorithm and proposes an entrepreneurial dynamic model based on improved machine learning. Aimed at the opportunistic behavior of the following venture capital institution under joint investment, this paper constructs an evolutionary game model between the leading venture capital institution and the following venture capital institution under joint investment and constructs an industrial cluster knowledge fusion and entrepreneurial innovation model. Moreover, this paper confirms through the structural equation model that the use of information fusion technology can improve the effectiveness and comprehensiveness of internal and external knowledge of industrial clusters. In addition, this paper uses experimental analysis methods to evaluate the performance of the entrepreneurial dynamic model constructed in this paper. From the research results, it can be seen that the system constructed in this paper has a certain effect.
\end{abstract}

\section{Introduction}

Today's economy has been dubbed a series of names such as knowledge economy, information economy, high-tech economy, and new economy, which is quite different from the previous economy characterized by low-intellectual labor. It is essentially an economy characterized by highly intelligent labor. The development of reality has increasingly confirmed Schumpeter's view that "the central issue of economics is not balance but structural change." Entrepreneurship will play a central role in this structural change. Moreover, the development of entrepreneurial activities has a positive effect on maintaining China's economic development, promoting a smooth transition of the economy, and smoothly realizing the adjustment and transformation of the economic structure [1].

In the context of global economic integration, how to avoid the impact of the external economy and how to overcome the limitations of the internal economic structure are major issues facing the sustainable development of our country's economy. Under the current situation, in order to be in line with the fundamental interests of economic development, the long-term stable development of China's economy must enable rapid and stable adjustments to the economic structure and gradually transform China's economic development mode. The emergence and development of the entrepreneurial economy are closely related to the knowledge economy, which is very different from the management economy. Labor, capital, and land are the main factors driving economic growth in the traditional management economy model; the core content of the entrepreneurial economy is to regard entrepreneurs and entrepreneurial spirit as an indispensable and important element of the economic form and also to promote economic development. The main factors can promote enterprise innovation and promote economic growth. In today's China, the role of entrepreneurial activities and new ventures in economic development has been recognized by all sectors of society. At the same time, research on entrepreneurs and entrepreneurial behavior has also become a hot topic in the field of 
entrepreneurship. With the progress of China's reform and opening up, the adjustment of the economic structure, the continuous deepening of the reform of state-owned enterprises, and the rapid rise of non-state-owned enterprises, new ventures have become an indispensable main component of China's national economy. As the largest developing country in the world, China's economic aggregate continues to grow, and its impact on the world economy is increasing. Improving the inherent quality of economic development and achieving steady and sustained economic growth are important issues that we face. It can be seen that entrepreneurial activities are of great significance to technological innovation, productivity improvement, employment increase, and national economic growth. This point has been recognized and confirmed by many scholars.

Entrepreneur research and the design and implementation of entrepreneurial support policies and measures have received great attention from countries and regions around the world, and entrepreneurship has become a global hot topic [2].

Based on this, this paper constructs a dynamic model of entrepreneurship with the support of improved machine learning algorithms. Through effective analysis of the model, the entrepreneurial process is studied, and on this basis, the efficiency of entrepreneurial analysis is further improved.

\section{Related Work}

Studies have shown that new ventures contribute to the growth of wealth, economic prosperity, and job creation in economic regions, thereby having a positive effect on the economic system [3]. With the implementation of entrepreneurial support policies, the scale of entrepreneurs is increasing, so how to identify entrepreneurs, how to distinguish the differences between entrepreneurs and nonentrepreneurs, and how entrepreneurs will develop have become topics of concern in the field of entrepreneurship research. Based on different entrepreneurial processes, the individual characteristics of entrepreneurs have different effects on entrepreneurial behavior. For example, in the entrepreneurial environment where the startup is a small- and medium-sized enterprise, the overall atmosphere and development direction of the entire startup will be directly affected by the entrepreneur's individual characteristics and spirit. Therefore, entrepreneurial research cannot ignore the research on the individual characteristics of entrepreneurs. The establishment and future development of new ventures will be affected by the individual characteristics of entrepreneurs. Therefore, the relationship between entrepreneurial characteristics and entrepreneurial inclination needs to be further studied.

Entrepreneurship process research is a hot spot in the field of entrepreneurship. Most of these studies focus on the analysis of resource development and value creation from the perspective of entrepreneurial team building, entrepreneurial opportunity identification, and entrepreneurial resource acquisition. Although the results of entrepreneurial research are becoming more and more abundant, there are not many studies on the identification of potential entrepreneurs and the antecedents of entrepreneurial behavior. The literature [4] points out that the antecedent factor of all entrepreneurial activities is entrepreneurial tendency. The research mainly focuses on the issue of entrepreneurial propensity and plans to deeply analyze the correlation between entrepreneurial antecedents, introduce path adjustment variables and intermediary variables, and finally establish a path model of entrepreneurial antecedents and entrepreneurial propensity.

Early entrepreneurial research described entrepreneurs as individuals with a high degree of confidence and risk taking in the face of uncertain and ambiguous situations and believed that entrepreneurs have distinct characteristics compared with nonentrepreneurs. Scholars believe that it is the inherent characteristics of individuals that make individuals take risky actions, which promote entrepreneurial behavior. Therefore, the research orientation of entrepreneurial characteristics tries to find the intrinsic characteristics of individuals that can identify entrepreneurs [5]. Entrepreneurs' individual characteristics, such as achievement needs, risk taking, internal control sources, and other individual psychological characteristics, have been confirmed by relevant empirical studies. This helps distinguish entrepreneurs from nonentrepreneurs and explain and predict the conversion of potential entrepreneurs to actual entrepreneurship. Among them, achievement demand is considered to be one of the most commonly used individual characteristic indicators that can successfully predict entrepreneurs [6]. However, some scholars believe that the research on entrepreneurial characteristics has not been able to explain in depth the internal causes of individuals participating in entrepreneurial activities [7]. Some scholars even question the research orientation of entrepreneurial characteristics and believe that the identification of entrepreneurs cannot be predicted only from individual characteristics [8]. The research on the cognitive process of entrepreneurship attempts to make up for the shortcomings of the research on entrepreneurial characteristics by exploring the cognitive factors that can stimulate entrepreneurship. The literature [9] believes that entrepreneurs and nonentrepreneurs have significant differences in the way of thinking and conducts research on the cognitive mechanisms that trigger entrepreneurs to identify entrepreneurial opportunities and engage in entrepreneurial activities. Moreover, it believes that from the perspectives of entrepreneurial cognitive mechanism, entrepreneurial decision-making types, entrepreneurial intentions, etc., it is possible to explore the important characteristics of entrepreneurs. Based on the study of the cognitive process of entrepreneurship, the literature [10] believes that the strength of individual entrepreneurial aspirations will be affected by entrepreneurial self-efficacy, and the essential factor that affects individual entrepreneurial behavior is individual entrepreneurial intentions. Individual entrepreneurial intention is the desire of an individual to actively prepare and strive to achieve the expected value of entrepreneurial behavior. The stronger this intention, the stronger its ability to predict actual entrepreneurial behavior.

The literature [11] points out that entrepreneurs are those who invent at the right time and transform their 
inventions into innovations, and it emphasizes the core role that entrepreneurs play in the innovation process. At the same time, these resource combinations established by entrepreneurs, including the supply of new raw materials, new product combinations, new production methods, new market development, and new organizational combinations, have become the guarantee for the implementation of economic reforms. The entrepreneurial process describes the process of a series of events triggered by the discovery of entrepreneurial opportunities and ultimately leading to the creation of a new enterprise [12]. The literature [13] points out that the description dimensions of the entrepreneurial process can be divided into three dimensions: the pursuit of profit in the market, the establishment of an organization, and the economic innovation. The literature [14] believes that there are three factors that will promote the entrepreneurial process, namely, entrepreneurs and entrepreneurial teams, entrepreneurial opportunities, and entrepreneurial resources. The literature [15] established a model for the establishment of new ventures. This mechanism model includes opportunity identification, commitment to the entrepreneurial process, production technology construction, organization establishment, product creation, market entry, and customer feedback. In order to feed back the abstract, driven, and actual entrepreneurial process, the mechanism model is repetitive and nonlinear. The framework developed in the literature [16] is based on the study of entrepreneurs and their enterprises and the sum of variables in previous studies. The individual variables of this framework include attitude, experience, and background. Although the use of psychological variables to study entrepreneurs was highly questioned before this, the three variables of achievement demand, control source, and risk tolerance are introduced into the research model of literature [17]. However, it can be seen from the four variables of the model that the process only enumerates some of the actions mentioned in the creation of new enterprises that are generally recognized in the society. The environment column mentions the various dimensions that will be involved in the social environment. The model in the literature [18] does not fundamentally fully explain the relationship between each column such as environment and process, individual and organization, or the other two, and there is even no relationship between variables.

\section{Model Solving and Stability Analysis}

The expected return of the leading venture capital institution $M$ to choose supervision and nonregulation is $U_{0}^{1}$ and $U_{0}^{2}$, respectively. The average return of the group of the leading venture capital institution $M$ is $\bar{U}^{0}$. According to the related theory of an evolutionary game [19],

$$
\begin{aligned}
& U_{0}^{1}=y\left[\alpha(R+\gamma \Delta V)-C_{0}\right]+(1-y)\left(\alpha R-C_{0}+\lambda R_{3}\right), \\
& U_{0}^{2}=y[\alpha(R+\Delta V)]+(1-y) \alpha R, \\
& \bar{U}_{0}=x U_{0}^{1}+(1-x) U_{0}^{2} .
\end{aligned}
$$

In the same way, the expected return of the following venture capital institution $N$ to choose active management and opportunism is $U_{1}^{1}$ and $U_{1}^{2}$, respectively. The average return of the group of the following venture capital institution $N$ is $\bar{U}_{1}$. According to the related theory of an evolutionary game,

$$
\begin{aligned}
& U_{1}^{1}=x[\beta(R+\gamma \Delta V)-C]+(1-x)[(\beta(R+\Delta V)-C)], \\
& U_{1}^{2}=x\left(\beta R+R_{1}-\lambda R_{2}\right)+(1-x)\left(\beta R+R_{1}\right), \\
& \bar{U}_{1}=y U_{1}^{1}+(1-y) U_{1}^{2} .
\end{aligned}
$$

According to the dynamic equation of replication, the growth rate of the leading venture capital institution and the following venture capital institution to choose supervision and active management, respectively, is

$$
\begin{aligned}
F(x) & =\frac{d x}{d t}=x\left(U_{0}^{1}-\bar{U}_{0}\right) \\
& =x(1-x)\left[y\left(\alpha \gamma \Delta V-\alpha \Delta V-\lambda R_{3}\right)+\left(\lambda R_{3}-C_{0}\right)\right]
\end{aligned}
$$

$$
\begin{aligned}
G(y) & =\frac{d y}{d t}=y\left(U_{1}^{1}-\bar{U}_{1}\right) \\
& =y(1-y)\left[x\left(\beta \gamma \Delta V-\beta \Delta V+\lambda R_{2}\right)+\left(\beta \Delta V-C-R_{1}\right)\right] .
\end{aligned}
$$

We set $d x / d t=0$ and $d y / d t=0$. When the replication dynamic equations of venture capital institutions $M$ and $N$ are set to zero, by solving the local equilibrium points of venture capital institutions $M$ and $N$ strategic interactions, five local equilibrium points of the system can be obtained, and they are, respectively, $(0,0),(0,1),(1,0),(1,1)$, and $\left(x_{0}, y_{0}\right)$. Among them,

$$
\begin{aligned}
& x_{0}=\frac{R_{1}+C-\beta \Delta V}{\beta \gamma \Delta V-\beta \Delta V+\lambda R_{2}}, \\
& y_{0}=\frac{C_{0}-\lambda R_{3}}{\alpha \gamma \Delta V-\alpha \Delta V-\lambda R_{3}} .
\end{aligned}
$$

When $0 \leq x_{0}, y_{0} \leq 1,\left(x_{0}, y_{0}\right)$ exists [20]

The Jacobian matrix of the joint investment strategy selection system is

$$
J=\left(\begin{array}{ll}
\frac{\partial F(x)}{\partial x} & \frac{\partial F(x)}{\partial y} \\
\frac{\partial G(x)}{\partial x} & \frac{\partial G(y)}{\partial y}
\end{array}\right)=\left(\begin{array}{ll}
a_{11} & a_{12} \\
a_{21} & a_{22}
\end{array}\right)
$$


Among them,

$a_{11}=(1-2 x)\left[y\left(\alpha \gamma \Delta V-\alpha \Delta V-\lambda R_{3}\right)+\left(\lambda R_{3}-C_{0}\right)\right]$,

$a_{12}=x(1-x)\left(\alpha \gamma \Delta V-\alpha \Delta V-\lambda R_{3}\right)$,

$a_{21}=y(1-y)\left(\beta \gamma \Delta V-\beta \Delta V+\lambda R_{2}\right)$,

$a_{22}=(1-2 y)\left[x\left(\beta \gamma \Delta V-\beta \Delta V+\lambda R_{2}\right)+\left(\beta \Delta V-C-R_{1}\right)\right]$.

Five partial equilibrium points are substituted into $a_{11}$, $a_{12}, a_{21}, a_{22}$ to get their specific values.

$$
\begin{aligned}
& Q=x_{0}\left(1-x_{0}\right)\left(\alpha \gamma \Delta V-\alpha \Delta V-\lambda R_{3}\right), \\
& R=y_{0}\left(1-y_{0}\right)\left(\beta \gamma \Delta V-\beta \Delta V+\lambda R_{2}\right) .
\end{aligned}
$$

To make the solution process simple, we set

$$
\begin{aligned}
& A=\lambda R_{3}-C_{0}, \\
& B=\alpha \gamma \Delta V-\alpha \Delta V-C_{0}, \\
& E=\beta \Delta V-C-R_{1}, \\
& F=\beta \gamma \Delta V+\lambda R_{2}-C-R_{1} .
\end{aligned}
$$

Because $\gamma \geq 1, \lambda \in(0,1), R_{2}>0, F>E$. At this time,

$$
\begin{aligned}
& x_{0}=\frac{-E}{F-E}, \\
& y_{0}=\frac{-A}{B-A} .
\end{aligned}
$$

$\left(x_{0}, y_{0}\right)$ is the center point. For leading venture capital institutions, if the initial state is $y=y_{0}$, then all $x$ are in a stable state. That is, when the proportion of the followtype venture capital institution choosing active management strategies is $y_{0}$, the proportion of leading venture capital institutions choosing supervision at any level can reach a stable state. When $y>y_{0}, F(x)>0, F^{\prime}(0)>0, F^{\prime}(1)<0$, according to the stability theorem of differential equations, $x=1$ is an evolutionary stable state. When $y<y_{0}, F(x)<0$, $F^{\prime}(0)<0, F^{\prime}(1)>0$, then $x=0$ is an evolutionary stable state [21].

For the following venture capital institutions, if the initial state is $x=x_{0}$, then all $y$ are in a stable state. That is, when the leading venture capital institution chooses a supervisory strategy ratio of $x_{0}$, the ratio of any level of the following venture capital institution choosing active management can reach a stable state. When $x>x_{0}, G(x)$ $>0, G^{\prime}(0)>0, G^{\prime}(1)<0$, according to the stability theorem of differential equations, $y=1$ is an evolutionary sta-

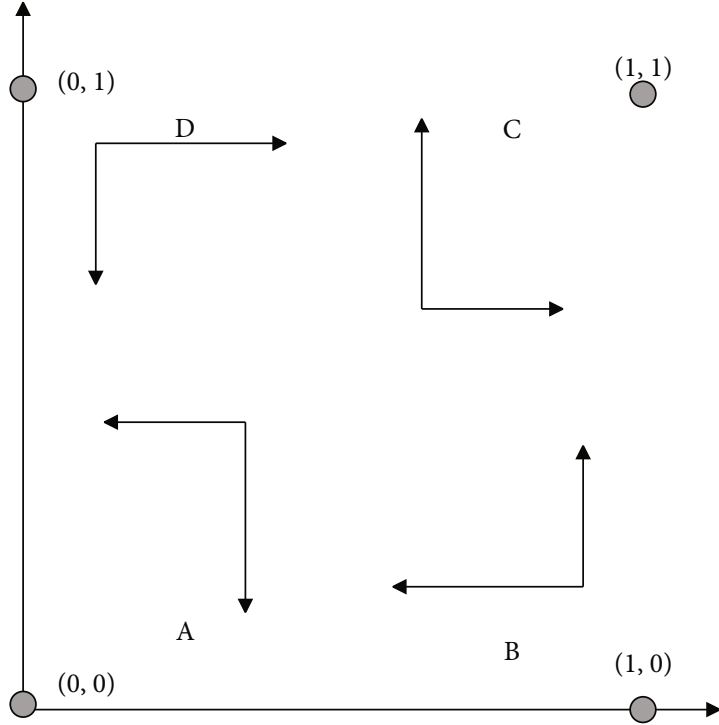

FIGURE 1: Phase diagram of the evolutionary game between the leading venture capital institution and the following venture capital institution.

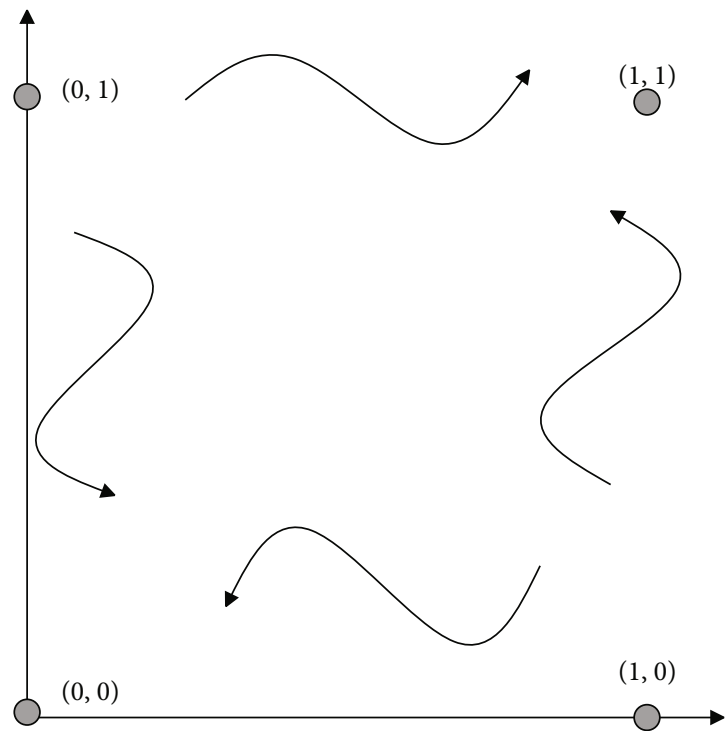

FIGURE 2: Evolution path diagram of the leading venture capital institution and the following venture capital institution.

ble state. When $x<x_{0}, G(x)<0, G^{\prime}(0)<0, G^{\prime}(1)>0, y=0$ is an evolutionary stable state.

According to the above stability analysis, the evolutionary game phase diagram shown in Figure 1 can be obtained.

According to the related theory of an evolutionary game and Figure 1, the evolution path diagram shown in Figure 2 can be obtained [22].

The final evolutionary stability strategy of the system is related to the initial state of the leading venture capital institution and the following venture capital institution. When the initial state of both parties falls in the OAEC area, the system will converge to point $O(0,0)$; that is, the leading venture capital institution chooses a nonregulatory strategy, 


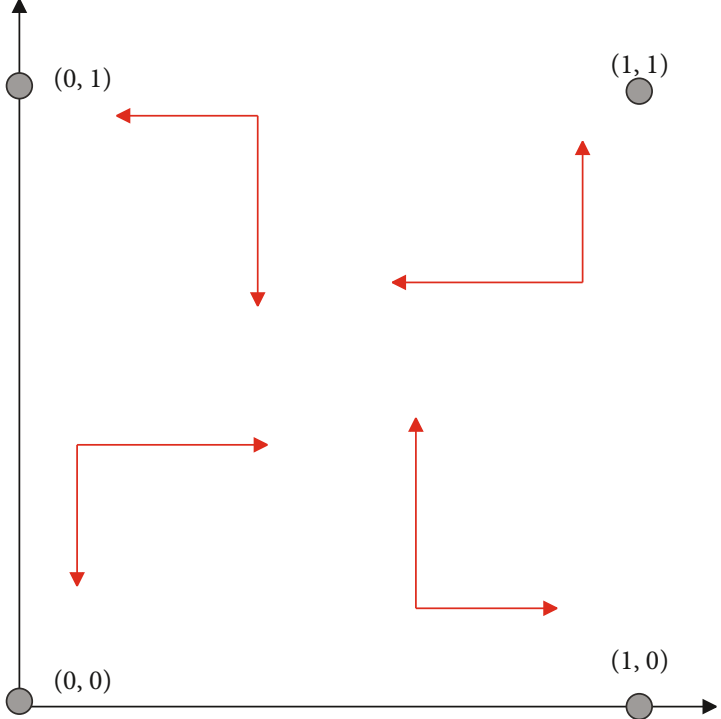

FIgURE 3: Evolution phase diagram of the leading venture capital institution and the following venture capital institution.

and the following venture capital institution chooses an opportunistic strategy. When the initial state of both parties falls in the $\mathrm{CEAB}$ area, the system will converge to point $B$ $(1,1)$; that is, the leading venture capital institution chooses a regulatory strategy, and the following venture capital institution chooses an active management strategy.

The following analysis is to solve the "prisoner's dilemma" that both parties tend to point $O$ and analyze which parameters will affect the final evolutionary stability strategy of both parties to converge to point $B$. Moreover, we analyzed which parameters will affect the area of the polygon CEAB from a geometrical point of view. The larger the $S_{\mathrm{CEAB}}$ is, the easier it is for the leading venture capital institution and the following venture capital institution to form a win-win situation of cooperation with supervision and active management [23].

$$
\begin{aligned}
S_{\mathrm{CEAB}}= & 1-S_{\mathrm{OCEA}}=1-\left(\frac{x_{0}}{2}+\frac{y_{0}}{2}\right) \\
= & 1-\frac{R_{1}+C-\beta \Delta V}{2\left(\beta \gamma \Delta V-\beta \Delta V+\lambda R_{2}\right)} \\
& -\frac{C_{0}-\lambda R_{3}}{2\left(\alpha \gamma \Delta V-\alpha \Delta V-\lambda R_{3}\right)} .
\end{aligned}
$$

(1) The influence of the profit ratio $\alpha, \beta$ of the leading venture capital institution and the following venture capital institution on the cooperation strategy is as follows:

$$
\begin{aligned}
& \frac{d S_{\mathrm{CEAB}}}{d \alpha}=\frac{\left(C_{0}-\lambda R_{3}\right)(\gamma \Delta V-\Delta V)}{2\left(\alpha \gamma \Delta V-\alpha \Delta V-\lambda R_{3}\right)^{2}}>0, \\
& \frac{d S_{\mathrm{CEAB}}}{d \beta}=\frac{\left(R_{1}+C-\beta \Delta V\right)(\gamma \Delta V-\Delta V)}{2\left(\beta \gamma \Delta V-\beta \Delta V-\lambda R_{2}\right)^{2}}>0 .
\end{aligned}
$$

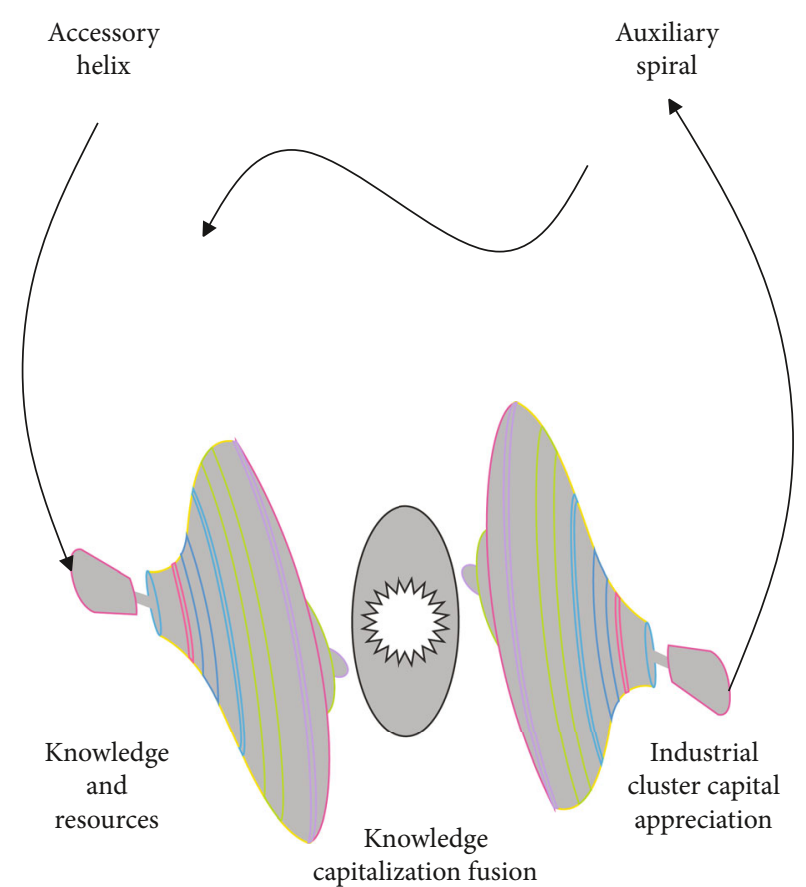

FIgURE 4: Process model of the knowledge capitalization of entrepreneurial talents to promote the technological upgrading of industrial clusters.

It can be seen that $S_{\mathrm{CEAB}}$ is positively correlated with the respective incomes of both parties. It shows that when other conditions remain the same, the greater the $\alpha, \beta$, the greater the probability that the initial state of the two parties will fall into the regional $\mathrm{CEAB}$, so that the leading venture capital institution and the following venture capital institution are more likely to form a cooperation and win-win situation of supervision and active management.

Conclusion: the greater the profit ratio of the leading venture capital institution and the following venture capital institution, the easier it is to form a cooperation situation.

(2) When the following venture capital institution chooses opportunistic behavior, the influence of probability $\lambda$ that is discovered on the cooperation strategy is as follows:

$$
\begin{aligned}
\frac{d S_{\mathrm{CEAB}}}{d \lambda}= & \frac{R_{2}\left(R_{1}+C-\beta \Delta V\right)}{2\left(\beta \gamma \Delta V-\beta \Delta V-\lambda R_{2}\right)^{2}} \\
& +\frac{R_{3}\left(\alpha \gamma \Delta V-\alpha \Delta V-\lambda R_{3}\right)-R_{3}\left(C_{0}-\lambda R_{3}\right)}{2\left(\alpha \gamma \Delta V-\alpha \Delta V-\lambda R_{3}\right)^{2}}>0 .
\end{aligned}
$$

It can be seen that $S_{\mathrm{CEAB}}$ is positively correlated with the probability $\lambda$ of being discovered when the following venture capital institution chooses opportunistic behavior. It shows that under the circumstance that other conditions remain the same, the greater the $\lambda$, the greater the probability that the initial state of both parties will fall into the regional 


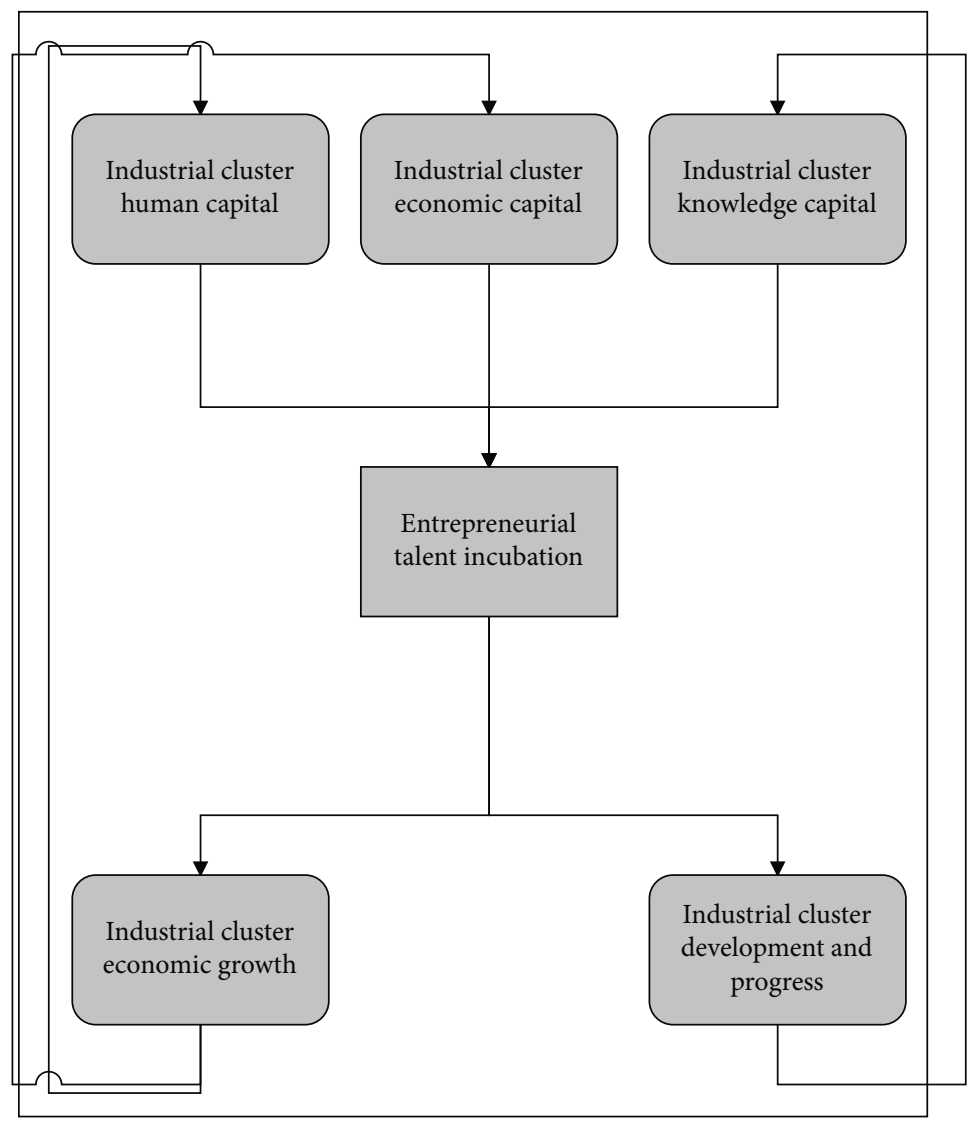

FIGURE 5: The structural model of the interaction model between entrepreneurial talents and industrial clusters.

$\mathrm{CEAB}$, so that the leading venture capital institution and the following venture capital institution are more likely to form a cooperation and win-win situation of supervision and active management.

Conclusion: the greater the probability of finding opportunistic behavior, the easier it is to form a cooperative situation.

(3) The influence of the synergy coefficient $\gamma$ of both parties on the cooperation strategy is as follows:

$\frac{d S_{\mathrm{CEAB}}}{d \gamma}=\frac{\beta \Delta V\left(R_{1}+C-\beta \Delta V\right)}{2\left(\beta \gamma \Delta V-\beta \Delta V-\lambda R_{2}\right)^{2}}+\frac{\alpha \Delta V\left(C_{0}-\lambda R_{3}\right)}{2\left(\alpha \gamma \Delta V-\alpha \Delta V-\lambda R_{3}\right)^{2}}>0$.

It can be seen that $S_{\mathrm{CEAB}}$ is positively correlated with the synergy coefficient $\gamma$ of both parties. It shows that under the condition that other conditions remain unchanged, the greater the $\gamma$, the greater the probability that the initial state of both parties will fall into the regional $\mathrm{CEAB}$, so that the leading venture capital institution and the following venture capital institution are more likely to form a cooperation and win-win situation of supervision and active management.

Conclusion: the greater the synergy coefficient between the leading venture capital institution and the following ven- ture capital institution, the easier it is to form a cooperation situation.

(4) The influence of the leading venture capital institution's regulatory costs $C_{0}$ on cooperation strategies and the leading venture capital institution is as follows:

$$
\frac{d S_{\mathrm{CEAB}}}{d C_{0}}=-\frac{1}{2\left(\alpha \gamma \Delta V-\alpha \Delta V-\lambda R_{3}\right)}<0 .
$$

It can be seen that $S_{\mathrm{CEAB}}$ is negatively related to the regulatory cost $C_{0}$ of the leading venture capital institution. It shows that under the circumstance that other conditions remain unchanged, the larger the $C_{0}$, the smaller the probability that the initial state of the two parties will fall in the regional $\mathrm{CEAB}$, so that the leading venture capital institution and the following venture capital institution are less likely to form a cooperation and win-win situation of supervision and active management.

Conclusion: the lower the cost of supervision of the leading venture capital institution, the easier it is to form a cooperation situation.

(5) The influence of the active management $\operatorname{cost} C$ of the following venture capital institution on the cooperation strategy is as follows: 


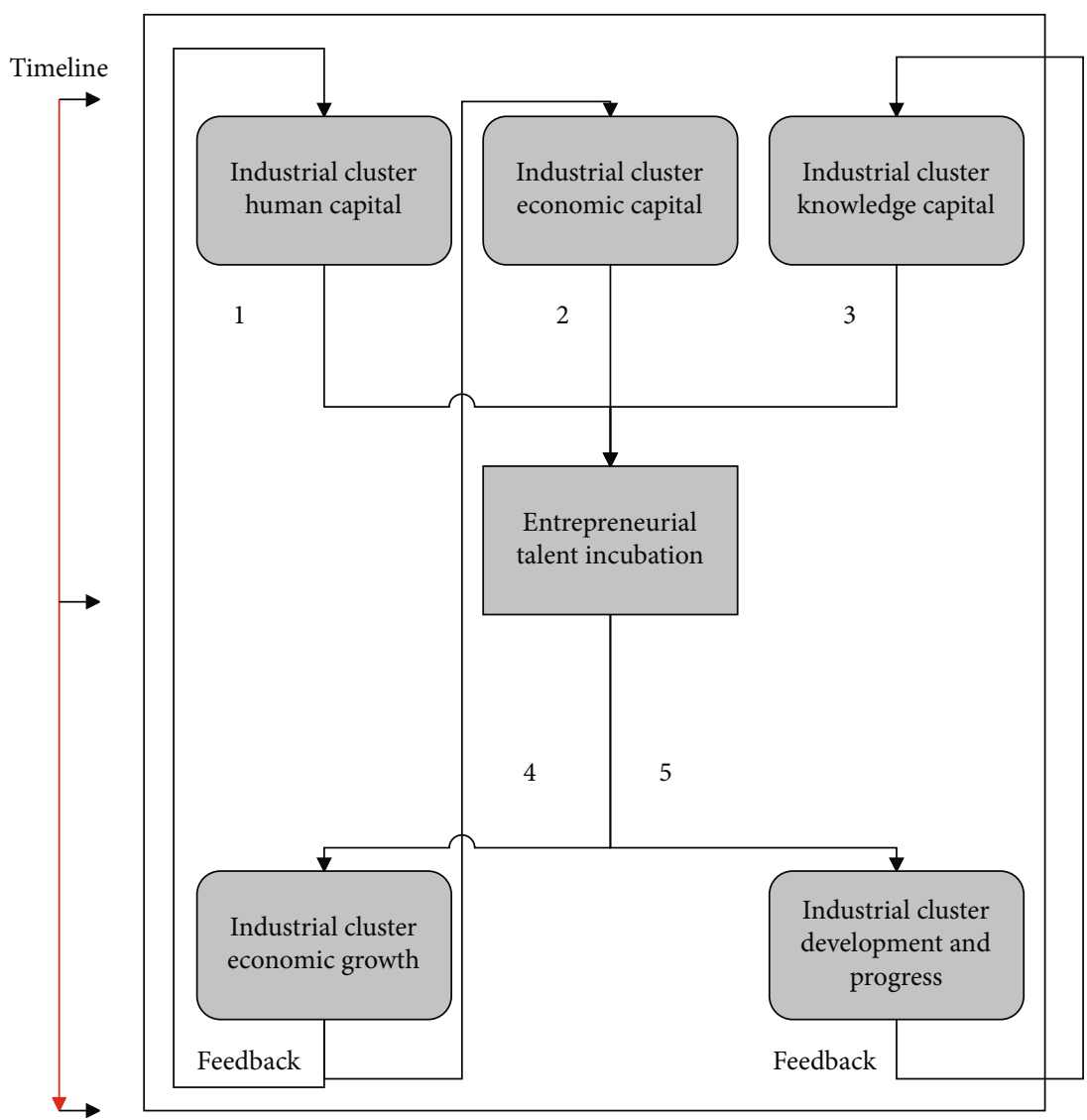

Figure 6: Test results of the interactive structure model between entrepreneurial talents and industrial clusters.

$$
\frac{d S_{\mathrm{CEAB}}}{d C}=-\frac{1}{2\left(\beta \gamma \Delta V-\beta \Delta V+\lambda R_{2}\right)}<0 .
$$

It can be seen that $S_{\mathrm{CEAB}}$ is negatively related to the active management cost $C$ of the following venture capital institution. It shows that under the circumstance that other conditions remain unchanged, the larger the $C$, the smaller the probability that the initial state of the two parties will fall in the regional $\mathrm{CEAB}$, so that the leading venture capital institution and the following venture capital institution are less likely to form a cooperation and win-win situation of supervision and active management.

Conclusion: the lower the active management cost of the following venture capital institution, the easier it is to form a cooperation situation.

(6) The influence of the following venture capital institution's additional profits $R_{1}$ brought by opportunistic behavior on the cooperation strategy is as follows:

$$
\frac{d S_{\mathrm{CEAB}}}{d R_{1}}=-\frac{1}{2\left(\beta \gamma \Delta V-\beta \Delta V+\lambda R_{2}\right)}<0 .
$$

It can be seen that $S_{\mathrm{CEAB}}$ is negatively correlated with the following venture capital institution's additional profits $R_{1}$ brought by opportunistic behavior. It shows that under the circumstance that other conditions remain unchanged, the larger the $R_{1}$, the smaller the probability that the initial state of the two parties will fall in the regional CEAB, so that the leading venture capital institution and the following venture capital institution are less likely to form a cooperation and win-win situation of supervision and active management.

Conclusion: the lower the additional benefits brought by opportunistic behavior, the easier it is to form a cooperative situation.

(7) The influence of the loss of profits $R_{2}$ on the cooperation strategy when the opportunistic behavior of the following venture capital institution is discovered is as follows:

$$
\frac{d S_{\mathrm{CEAB}}}{d R_{2}}=-\frac{1}{2\left(\beta \gamma \Delta V-\beta \Delta V+\lambda R_{2}\right)}>0 .
$$

It can be seen that $S_{\mathrm{CEAB}}$ is positively correlated with the loss of profits $R_{2}$ when the following venture capital institution's opportunistic behavior is discovered. It shows that under the circumstance that other conditions remain unchanged, the greater the $R_{2}$, the greater the probability that the initial state of both parties will fall into the regional $\mathrm{CEAB}$, so that the leading venture capital institution and the following venture capital institution are more likely to form 


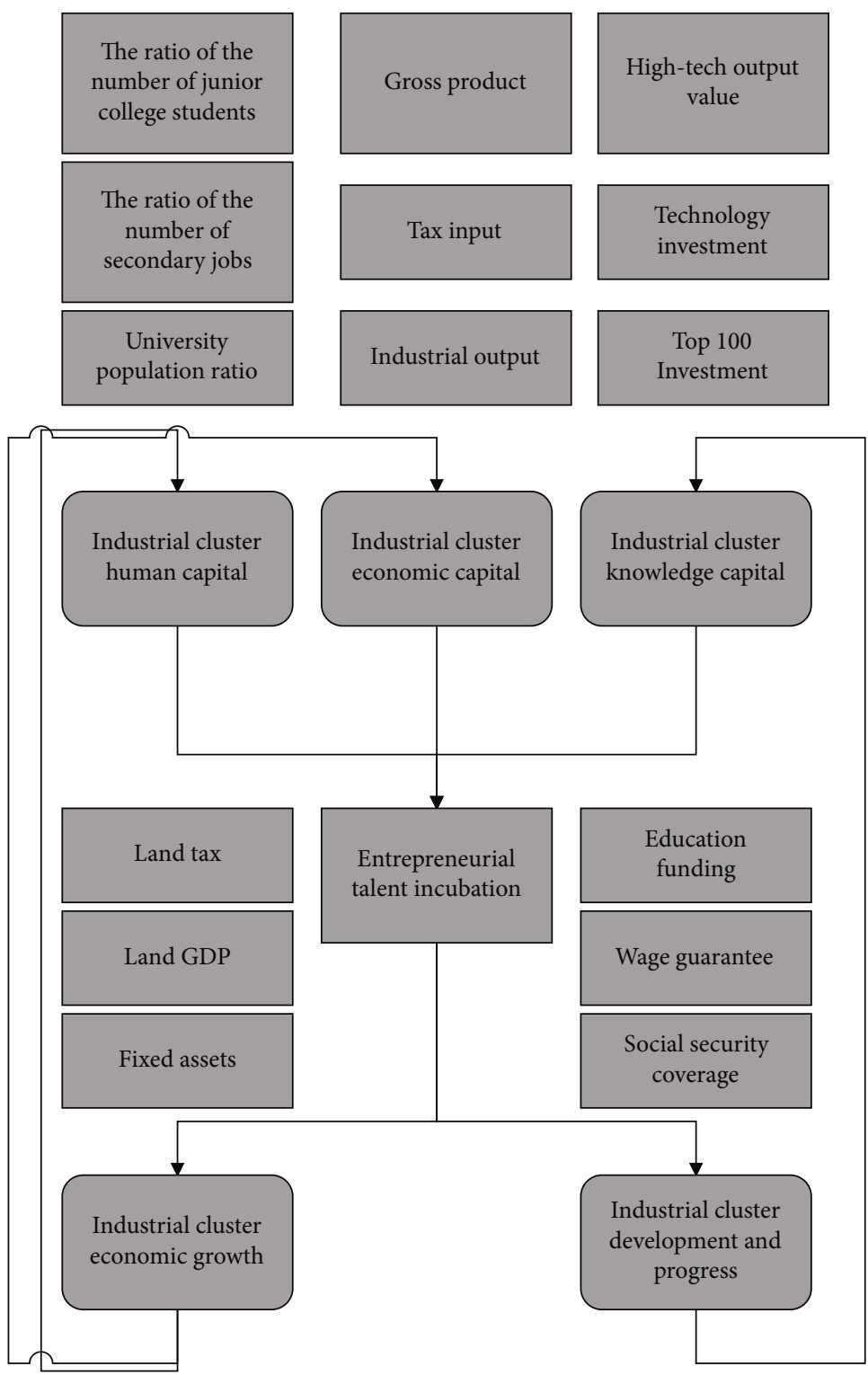

Figure 7: Simulation structure model.

a cooperation and win-win situation of supervision and active management.

Conclusion: the greater the loss when the opportunistic behavior of the following venture capital institution is discovered, the easier it is to form a situation of cooperation.

(8) The influence of fines $R_{3}$ collected by the leading venture capital institutions for discovering opportunistic behaviors of the follow-type venture capital institution on cooperation strategies is as follows

$$
\frac{d S_{\mathrm{CEAB}}}{d R_{3}}=\frac{\lambda\left(\alpha \gamma \Delta V-\alpha \Delta V-\lambda R_{3}\right)-\lambda\left(C_{0}-\lambda R_{3}\right)}{2\left(\alpha \gamma \Delta V-\alpha \Delta V-\lambda R_{3}\right)^{2}}>0 .
$$

It can be seen that $S_{\mathrm{CEAB}}$ is positively correlated with the fine $R_{3}$ collected by the leading venture capital institution for discovering opportunistic behaviors of the following venture capital institution. It shows that under the circumstance that other conditions remain the same, the larger the $R_{3}$, the greater the probability that the initial state of the two parties will fall into the regional CEAB, and thus, the leading venture capital institution and the following venture capital institution are more likely to form a cooperation and win-win situation of supervision and active management.

Conclusion: the more fines the leading venture capital institution charges for the following opportunistic behavior, the easier it is to form a cooperative situation. 
TABLE 1: Statistical table of the effect of the entrepreneurial dynamic model on data processing of entrepreneurial factors.

\begin{tabular}{|c|c|c|c|c|c|}
\hline No. & Entrepreneurship data analysis & No. & Entrepreneurship data analysis & No. & Entrepreneurship data analysis \\
\hline 1 & 92.7 & 30 & 86.4 & 59 & 86.7 \\
\hline 2 & 84.3 & 31 & 92.8 & 60 & 79.7 \\
\hline 3 & 87.8 & 32 & 93.6 & 61 & 92.5 \\
\hline 4 & 81.3 & 33 & 80.8 & 62 & 87.4 \\
\hline 5 & 84.0 & 34 & 83.3 & 63 & 84.3 \\
\hline 6 & 80.3 & 35 & 88.8 & 64 & 81.1 \\
\hline 7 & 84.9 & 36 & 84.0 & 65 & 88.7 \\
\hline 8 & 90.0 & 37 & 89.4 & 66 & 88.5 \\
\hline 9 & 83.5 & 38 & 90.3 & 67 & 80.6 \\
\hline 10 & 92.0 & 39 & 82.4 & 68 & 87.5 \\
\hline 11 & 79.6 & 40 & 79.8 & 69 & 89.3 \\
\hline 12 & 86.9 & 41 & 80.0 & 70 & 87.0 \\
\hline 13 & 85.0 & 42 & 84.9 & 71 & 82.8 \\
\hline 14 & 86.9 & 43 & 82.1 & 72 & 90.1 \\
\hline 15 & 92.9 & 44 & 92.6 & 73 & 79.3 \\
\hline 16 & 89.0 & 45 & 87.3 & 74 & 90.9 \\
\hline 17 & 93.2 & 46 & 86.7 & 75 & 93.2 \\
\hline 18 & 91.7 & 47 & 84.7 & 76 & 90.5 \\
\hline 19 & 90.4 & 48 & 87.2 & 77 & 80.7 \\
\hline 20 & 81.9 & 49 & 84.8 & 78 & 88.9 \\
\hline 21 & 87.4 & 50 & 82.8 & 79 & 92.7 \\
\hline 22 & 84.8 & 51 & 93.3 & 80 & 91.8 \\
\hline 23 & 92.4 & 52 & 92.2 & 81 & 93.3 \\
\hline 24 & 80.5 & 53 & 82.3 & 82 & 82.5 \\
\hline 25 & 81.5 & 54 & 90.7 & 83 & 81.1 \\
\hline 26 & 87.1 & 55 & 93.1 & 84 & 88.4 \\
\hline 27 & 92.2 & 56 & 90.6 & 85 & 88.3 \\
\hline 28 & 83.8 & 57 & 82.8 & 86 & 80.8 \\
\hline 29 & 83.3 & 58 & 86.0 & 87 & 80.9 \\
\hline
\end{tabular}

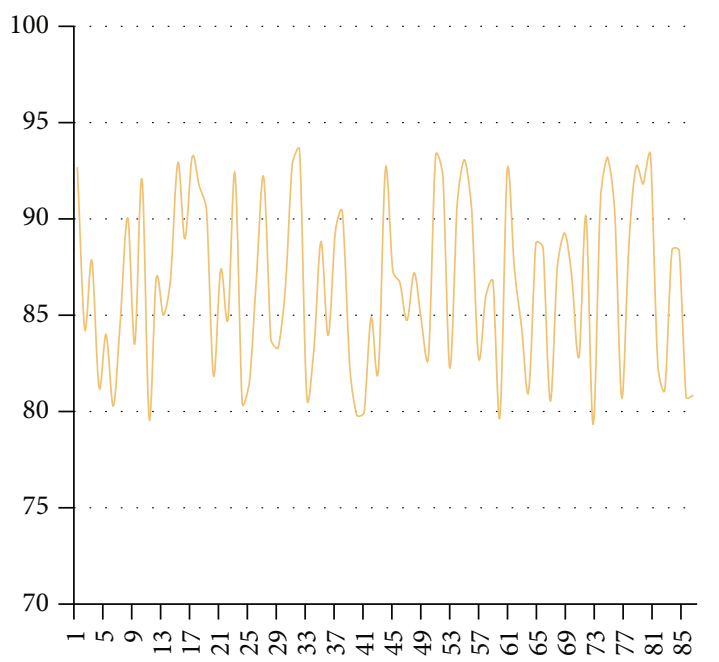

FIGURE 8: Statistical diagram of the effect of the entrepreneurial dynamic model on data processing of entrepreneurial factors.
In condition 7 , the system has no evolutionary stable solution. The evolutionary game phase diagram is shown in Figure 3.

It can be seen from Figure 3 that there is no evolutionary stable solution in the four quadrants. Therefore, the entire system will be in constant change.

\section{Entrepreneurship Dynamic Model Construction}

Entrepreneurial talents gather the knowledge competitiveness of industrial clusters, transform capital competitiveness, and promote the development of industrial clusters in a process similar to the way in which auxiliary spirals and auxiliary spirals function in the natural world. There are two kinds of spirals in meteorology. One kind of spirals accumulates matter centripetally and gathers a large amount of energy at the tip of the spiral to form matter fusion. This is called auxiliary spiral; the other is to separate matter and energy. The spiral cone tip is released through the 
TABLE 2: Statistical table of the entrepreneurial analysis effect of the entrepreneurial dynamic model.

\begin{tabular}{|c|c|c|c|c|c|}
\hline No. & Entrepreneurial effect & No. & Entrepreneurial effect & No. & Entrepreneurial effect \\
\hline 1 & 69.4 & 30 & 82.5 & 59 & 73.6 \\
\hline 2 & 84.4 & 31 & 83.0 & 60 & 89.5 \\
\hline 3 & 79.1 & 32 & 77.9 & 61 & 69.6 \\
\hline 4 & 84.9 & 33 & 82.3 & 62 & 74.1 \\
\hline 5 & 88.1 & 34 & 90.8 & 63 & 69.0 \\
\hline 6 & 72.0 & 35 & 86.7 & 64 & 72.2 \\
\hline 7 & 68.8 & 36 & 72.6 & 65 & 82.0 \\
\hline 8 & 68.5 & 37 & 88.1 & 66 & 75.4 \\
\hline 9 & 80.8 & 38 & 71.3 & 67 & 79.9 \\
\hline 10 & 73.6 & 39 & 89.6 & 68 & 68.2 \\
\hline 11 & 72.4 & 40 & 69.4 & 69 & 74.0 \\
\hline 12 & 85.5 & 41 & 76.7 & 70 & 71.6 \\
\hline 13 & 76.8 & 42 & 73.7 & 71 & 69.2 \\
\hline 14 & 85.4 & 43 & 80.0 & 72 & 80.0 \\
\hline 15 & 74.7 & 44 & 69.0 & 73 & 86.5 \\
\hline 16 & 72.8 & 45 & 71.5 & 74 & 74.7 \\
\hline 17 & 84.5 & 46 & 78.2 & 75 & 75.7 \\
\hline 18 & 86.5 & 47 & 87.8 & 76 & 90.4 \\
\hline 19 & 70.8 & 48 & 77.7 & 77 & 69.3 \\
\hline 20 & 68.9 & 49 & 77.3 & 78 & 76.6 \\
\hline 21 & 69.0 & 50 & 89.6 & 79 & 77.5 \\
\hline 22 & 87.3 & 51 & 72.7 & 80 & 83.0 \\
\hline 23 & 72.5 & 52 & 76.8 & 81 & 83.2 \\
\hline 24 & 86.3 & 53 & 68.8 & 82 & 86.1 \\
\hline 25 & 70.6 & 54 & 77.2 & 83 & 85.9 \\
\hline 26 & 83.5 & 55 & 86.3 & 84 & 85.2 \\
\hline 27 & 85.2 & 56 & 83.2 & 85 & 68.6 \\
\hline 28 & 86.8 & 57 & 89.7 & 86 & 80.2 \\
\hline 29 & 77.0 & 58 & 83.5 & 87 & 78.3 \\
\hline
\end{tabular}

centrifugal rotation orbit, which is called the auxiliary scattered spiral.

There is no pure auxiliary spiral or pure auxiliary spiral in nature. If there is material or energy spiraling in from the periphery of the spiral, there must be an equal amount of energy spiraling out from the tip of the spiral. It is the focus of qualitative energy and the gate of qualitative change of matter. This phenomenon is similar to the process of entrepreneurial talent incubation to promote the development of industrial clusters. In the interactive model of industrial clusters and entrepreneurial talents, the two precession spirals of industrial cluster development and entrepreneurial talent incubation share a spiral tip, in which entrepreneurial talents accumulate knowledge. It forms a complementary spiral with resources and realizes the capitalization transformation at the tip of the cone. After the capitalization transformation, knowledge and resources can be more widely recognized by other individuals and then form a complementary spiral through a wide range of knowledge spillovers within the industrial cluster. Finally, it spreads among other individuals in the cluster and drives the overall development of the cluster. The developed cluster can better support the accumulation of material and energy for entrepreneurial talents to generate a complementary spiral.

The process model is shown in Figure 4.

In order to more clearly define regional development and progress and economic growth, this study combines existing empirical research in this area and summarizes the promotion of entrepreneurial talents on industrial clusters into two aspects: capital appreciation and noncapital factors. In terms of entrepreneurial talents regarding the economic growth of regional development, entrepreneurial talent is a group that seeks to maximize profits and can discover the profit space and entrepreneurial opportunities in the market. In terms of noncapital factors (economic development), entrepreneurial talent can drive the region. The selfrealization process of internal individuals brings new knowledge and technology to industrial clusters, promotes the diversified development of market technology, and promotes regional knowledge spillover and technology diffusion. A thorough consideration of these two aspects can systematize the relationship between entrepreneurial talents 


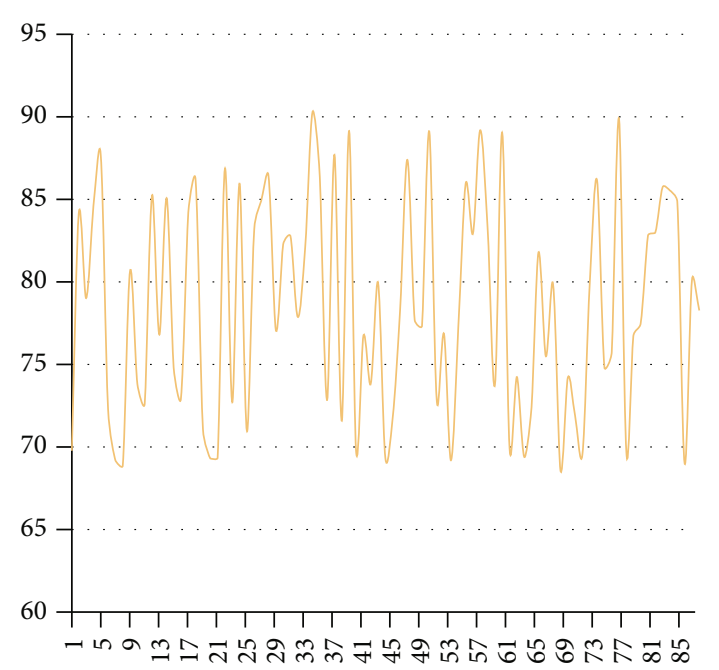

Figure 9: Statistical diagram of the entrepreneurial analysis effect of the entrepreneurial dynamic model.

and the development of industrial clusters. Hypothesis a: there is a positive correlation between entrepreneurial talent incubation and the economic growth of high-tech industrial clusters. Hypothesis b: there is a positive correlation between entrepreneurial talent incubation and the economic growth of traditional industrial clusters. Hypothesis 2a: there is a positive relationship between entrepreneurial talent incubation and the development and progress of high-tech industrial clusters. Correlation hypothesis $2 \mathrm{~b}$ : there is a positive correlation between entrepreneurial talent incubation and the development and progress of traditional industrial clusters.

The interactive system of entrepreneurial talents and industrial clusters includes three components. The first is the economic capital, human capital, and knowledge capital of industrial clusters. This level of factors constitutes the foundation for the incubation of entrepreneurial talents. The second layer is the incubation of entrepreneurial talents, and the third layer is the economic growth of industrial clusters and the overall development and progress of industrial clusters. The model construction is shown in Figure 5.

Through the machine learning algorithm constructed in this paper, the size of the entrepreneurial equilibrium value and the relationship between the entrepreneurial equilibrium value and the economic development level of the industrial cluster can be obtained. It can be seen that hypothesis a passes the test and hypothesis b fails the test. Therefore, the test result of the interactive structure model of entrepreneurial talents and industrial clusters is shown in Figure 6.

After the input and output variables are determined, this research studies the construction model of the double helix interaction hypothesis between industrial clusters and entrepreneurial talents. Since previous studies have also pointed out that the contribution of entrepreneurial talents varies from region to region with different development models, and industrial clusters with different development models have different influencing factors when interacting with entrepreneurial talents. Therefore, before proceeding with the interaction hypothesis, this study classifies the development models of industrial clusters. The simulation structure model diagram is shown in Figure 7.

\section{Performance Verification of the Entrepreneurial Dynamic Model Based on Improved Machine Learning}

This paper constructs the entrepreneurial dynamic model based on the improved machine learning algorithm and evaluates the performance of the constructed system after constructing the entrepreneurial dynamic model. Since the entrepreneurial dynamic model requires a large number of entrepreneurial factor analysis data, this paper inputs a large number of factor analysis data through simulation software, and the results obtained are shown in Table 1 and Figure 8.

From the above analysis, it can be seen that the system constructed in this paper has a stable performance in the data analysis of entrepreneurial factors. On this basis, the entrepreneurial analysis effect of the entrepreneurial dynamic model is evaluated. The results are shown in Table 2 and Figure 9.

From the above analysis results, it can be seen that the entrepreneurial dynamic model constructed in this paper can basically meet the basic needs of entrepreneurial analysis.

\section{Conclusion}

The economic environment, entrepreneurial conditions, and the stock and flow of intellectual capital of industrial clusters are important determinants that affect the incubation of entrepreneurial talents. Previously, many scholars conducted research on entrepreneurial talents in the context of the country, rather than in specific industrial clusters. This paper combines improved machine learning algorithms to construct a dynamic model of entrepreneurship. Moreover, in response to the opportunistic behavior of following venture capital institutions under joint investment, this paper constructs an evolutionary game model between leading venture capital institutions and following venture capital institutions under joint investment. In addition, by solving the dynamic replication equation and the evolutionary stability strategy, this paper constructs the system model according to the actual investment situation. Finally, after constructing the model, this article analyzes the system performance through simulation. From the research and results, it can be seen that the entrepreneurial dynamic model constructed in this article has a certain effect.

\section{Data Availability}

The labeled dataset used to support the findings of this study are available from the corresponding author upon request.

\section{Conflicts of Interest}

The authors declare no competing interests. 


\section{Acknowledgments}

This study is sponsored by 2020 Special Project of Humanities and Social Sciences of Shaanxi Provincial Education Department: "Research on the Evaluation of Innovation and Entrepreneurship Ecosystem Based on Grey Association Law - Take Xi'an City as an Example" (20JK0046).

\section{References}

[1] J. Albrecht, M. Robayo-Abril, and S. Vroman, "Public-sector employment in an equilibrium search and matching model," The Economic Journal, vol. 129, no. 617, pp. 35-61, 2019.

[2] P. Barbieri, R. Bozzon, S. Scherer, R. Grotti, and M. Lugo, "The rise of a Latin model? Family and fertility consequences of employment instability in Italy and Spain," European Societies, vol. 17, no. 4, pp. 423-446, 2015.

[3] C. Barnard, "EU employment law and the European social model: the past, the present and the future," Current Legal Problems, vol. 67, no. 1, pp. 199-237, 2014.

[4] G. R. Bond and R. E. Drake, "Making the case for IPS supported employment," Administration and Policy in Mental Health and Mental Health Services Research, vol. 41, no. 1, pp. 69-73, 2014.

[5] G. R. Bond, S. J. Kim, D. R. Becker et al., "A controlled trial of supported employment for people with severe mental illness and justice involvement," Psychiatric Services, vol. 66, no. 10, pp. 1027-1034, 2015.

[6] M. Bornay-Barrachina, A. López-Cabrales, and R. Valle-Cabrera, "How do employment relationships enhance firm innovation? The role of human and social capital," The InTernaTIonal Journal of human resource managemen $T$, vol. 28, no. 9, pp. 1363-1391, 2017.

[7] J. H. Fede, K. S. Gorman, and M. E. Cimini, "Student employment as a model for experiential learning," The Journal of Experimental Education, vol. 41, no. 1, pp. 107-124, 2018.

[8] P. Frijters, D. W. Johnston, and M. A. Shields, "The effect of mental health on employment: evidence from Australian panel data," Health Economics, vol. 23, no. 9, pp. 1058-1071, 2014.

[9] U. Huws, N. H. Spencer, and D. S. Syrdal, "Online, on call: the spread of digitally organised just-in-time working and its implications for standard employment models," New Technology, Work and Employment, vol. 33, no. 2, pp. 113-129, 2018.

[10] F. Latorre, D. Guest, J. Ramos, and F. J. Gracia, "High commitment HR practices, the employment relationship and job performance: a test of a mediation model," European Management Journal, vol. 34, no. 4, pp. 328-337, 2016.

[11] C. C. Lo and T. C. Cheng, "Race, employment disadvantages, and heavy drinking: a multilevel model," Journal of Psychoactive Drugs, vol. 47, no. 3, pp. 221-229, 2015.

[12] L. Lynas, "Project ABLE (autism: building links to employment): a specialist employment service for young people and adults with an autism spectrum condition," Journal of Vocational Rehabilitation, vol. 41, no. 1, pp. 13-21, 2014.

[13] T. Marshall, R. W. Goldberg, L. Braude et al., "Supported employment: assessing the evidence," Psychiatric Services, vol. 65, no. 1, pp. 16-23, 2014.

[14] C. Dillahunt-Aspillaga, R. Nakase-Richardson, T. Hart et al., "Predictors of employment outcomes in veterans with traumatic brain injury: a VA traumatic brain injury model systems study," Journal of Head Trauma Rehabilitation, vol. 32, no. 4, pp. 271-282, 2017.

[15] L. Ottomanelli, S. Barnett, L. Goetz, and R. Toscano, "Vocational rehabilitation in spinal cord injury: what vocational service activities are associated with employment program Outcome?," Topics in Spinal Cord Injury Rehabilitation, vol. 21, no. 1, pp. 31-39, 2015.

[16] F. Portella-Carbo, "Effects of international trade on domestic employment: an application of a global multiregional inputoutput supermultiplier model (1995-2011)," Economic Systems Research, vol. 28, no. 1, pp. 95-117, 2016.

[17] M. Shirmohammadi, M. Beigi, and J. Stewart, "Understanding skilled migrants' employment in the host country: a multidisciplinary review and a conceptual model," The International Journal of Human Resource Management, vol. 30, no. 1, pp. 96-121, 2019.

[18] D. Tavani and L. Zamparelli, "Endogenous technical change, employment and distribution in the Goodwin model of the growth cycle," Studies in Nonlinear Dynamics \& Econometrics, vol. 19, no. 2, pp. 209-216, 2015.

[19] G. Topa, C. M. Alcover, J. A. Moriano, and M. Depolo, "Bridge employment quality and its impact on retirement adjustment: a structural equation model with SHARE panel data," Economic and Industrial Democracy, vol. 35, no. 2, pp. 225-244, 2014.

[20] C. Topsakal, "Policy on teacher employment model in Turkey: contracted teachers," International Journal of Educational Methodology, vol. 5, no. 4, pp. 671-682, 2019.

[21] E. Totty, "The effect of minimum wages on employment: a factor model approach," Economic Inquiry, vol. 55, no. 4, pp. 1712-1737, 2017.

[22] S. Van de Walle, B. Steijn, and S. Jilke, "Extrinsic motivation, PSM and labour market characteristics: a multilevel model of public sector employment preference in 26 countries," International Review of Administrative Sciences, vol. 81, no. 4, pp. 833-855, 2015.

[23] J. F. Wen and D. V. Gordon, "An empirical model of tax convexity and self-employment," Review of Economics and Statistics, vol. 96, no. 3, pp. 471-482, 2014. 